\title{
Diet, Gut Microbiota, and Vitamins D + A in Multiple Sclerosis
}

\author{
Paolo Riccio $^{1}$ (D) Rocco Rossano $^{1}$
}

Published online: 24 October 2017

(C) The American Society for Experimental NeuroTherapeutics, Inc. 2017

\begin{abstract}
Central to the understanding of the relationships between diet, gut microbiota, and vitamins $\mathrm{D}$ and $\mathrm{A}$ in multiple sclerosis is low-grade inflammation, which is involved in all chronic inflammatory diseases and is influenced by each of the above effectors. We show that food components have either proinflammatory or anti-inflammatory effects and influence both the human metabolism (the "metabolome") and the composition of gut microbiota. Hypercaloric, high-animal-fat Western diets favor anabolism and change gut microbiota composition towards dysbiosis. Subsequent intestinal inflammation leads to leakage of the gut barrier, disruption of the blood-brain barrier, and neuroinflammation. Conversely, a vegetarian diet, rich in fiber, is coherent with gut eubiosis and a healthy condition. Vitamin D levels, mainly insufficient in a persistent low-grade inflammatory status, can be restored to optimal values only by administration of high amounts of cholecalciferol. At its optimal values ( $>30 \mathrm{ng} / \mathrm{ml})$, vitamin D requires vitamin $\mathrm{A}$ for the binding to the vitamin $\mathrm{D}$ receptor and exert its anti-inflammatory action. Both vitamins must be supplied to the subjects lacking vitamin D. We conclude that nutrients, including the nondigestible dietary fibers, have a leading role in tackling the low-grade inflammation associated with chronic inflammatory diseases. Their action is mediated by gut microbiota and any microbial change induced by diet
\end{abstract}

Electronic supplementary material The online version of this article (https://doi.org/10.1007/s13311-017-0581-4) contains supplementary material, which is available to authorized users.

Paolo Riccio

paoloxriccio@gmail.com

1 Department of Sciences, University of Basilicata, Viale dell'Ateneo Lucano, 10, 85100 Potenza, Italy modifies host-microbe interactions in a consequent way, to improve the disease or worsen it.

Keywords Diet $\cdot$ Gut Microbiota $\cdot$ Vitamin D $\cdot$ Multiple Sclerosis $\cdot$ Neuroinflammation

\section{Introduction}

Multiple sclerosis (MS), a debilitating disease that affects young adults, especially women, is a chronic demyelinating and neurodegenerative disease of an inflammatory and autoimmune nature, whose etiopathogenesis is not yet known.

According to Compston and Coles [1], "Multiple sclerosis is primarily an inflammatory disorder of the brain and spinal cord in which focal lymphocytic infiltration leads to damage of myelin and axons".

This definition of MS is useful to highlight that inflammation or, more precisely low-grade inflammation, is the primary target to address in order to ameliorate the course of the disease [2]. Here we show that there is a direct link between human dietary behaviors, gut microbiota, vitamin $\mathrm{D}$, and MS (including other inflammatory neurological disorders), and that nutritional intervention and administration of vitamins D and A (in the case of hypovitaminosis D) may be effective tools with which to dampen low-grade inflammation.

What is the evidence for a role of diet and gut microbiota in the low-grade inflammation associated with MS?

MS is a multifactorial disease and various environmental factors may have a role in its development [3]. Environmental factors are of 2 types: a) of nondietetic origin: 1) viral infections (no association with Epstein-Barr virus was established in the BENEFIT study [4]); 2) heavy metal poisoning [5]; 3 ) cigarette smoke (no association with tobacco was 
established in the BENEFIT study [4]); b) associated with dietary habits and metabolic conditions: 1) excess body weight in childhood and adolescence $[6,7]$; 2) low vitamin D status [8,9]; 3) sedentary lifestyle and high-animal-fat/highsugar Western diets [10-13].

However, there are some indications suggesting that dietary habits and lifestyle, rather than infections or nondietary factors, may influence the course of disease: 1) The geographical distribution of MS is more prevalent in Western countries at northern latitudes, i.e., with lower exposure to sunlight. However, as the disease is not widespread in Eastern countries at the corresponding latitudes, what makes the difference is the sedentary lifestyle and the energy-dense dietary habits of Western countries, not the lower availability of vitamin $\mathrm{D}$, which seems to be widespread worldwide [14]. 2) Western diets are associated with postprandial inflammation [15]. 3) High body mass index (BMI) before the age of 20 years is associated with $2 x$ increased risk [16].

The question of whether food is actually related to lowgrade inflammation and how it can control it arises. This is fundamental in our context because inflammatory injuries are major drivers of neural damage in MS [2]. In this regard, it is possible to affirm that specific dietary factors may have either proinflammatory or anti-inflammatory effects and that these effects are exerted directly on our metabolism or indirectly through their action on gut microbiota [10-13].

In fact, gut microbiota can influence our metabolism and our inflammatory state. Moreover, through the gut-brain axis, it can also affect the condition of the central nervous system in health and disease [17-19].

Gut dysbiosis may be associated with endotoxemia, intestinal/systemic inflammation, and trigger neuroinflammation, whereas a healthy gut microbiota may dampen the inflammatory processes with the production of antiinflammatory molecules.

This review is therefore about the relationships between nutrition, gut microbiota, low-grade inflammation, and MS. This is an important task, because, at present, MS therapy is not associated with a particular diet, probably owing to lack of information on the effects of nutrition on the disease.

\section{Eating: A Normal Daily Act}

\section{The Food-Human Metabolome-Gut Microbiota Axis}

According to the World Health Organization, "nutrition is the intake of food, considered in relation to the body's dietary needs". Actually, in order for food intake to become nutritious, it must be digested into simple units, then absorbed and used to provide energy or build the molecules necessary for growth, repair, and survival. This process, called nutrition, is mandatory and physiologic. Normally, food, taken in the right amount, does not cause damage to the host, given that it is natural, not processed, not adulterated, and well digested. However, as dietary habits may be proinflammatory or antiinflammatory, the choice of proinflammatory diets can lead to the human chronic inflammatory diseases typical of our time [11-13]. This may happen because food is not simply a matter of calories but influences the metabolism of the recipients. The disclosed recipients are basically 2: our metabolome and our gut microbiota. In this food-metabolome-gut axis the relationship is reciprocal: both the host and its gut microbiota metabolize their nutrients and are, in turn, transformed by them. Changes in our metabolism and in microbiota population due to our dietary habits can improve or worsen our health.

When evaluating the impact of nutrition on human health, let us take into consideration food intake beforehand: this may differ for quality, i.e., the composition of diet (what we eat), and quantity, i.e., the amount of food intake (how much we eat).

With regard to the first point (what we eat), this is relevant because food may contain dietary factors capable of activating or inhibiting nuclear receptors, transcription factors, or enzymes, which, in turn, regulate the expression of specific genes and thereby drive metabolic pathways towards either catabolism or anabolism. In principle, catabolism is associated with a healthy status, whereas anabolism may be associated with or may predispose to inflammatory processes [13].

With regard to the second point (how much we eat), the influence of food is mainly indirect. Fasting or caloric restriction, even at times, are more appropriate for a healthy status, as they stimulate catabolism and inhibit anabolism and lowgrade inflammation, whereas hypercaloric, high-fat diets show the opposite effects. It should be pointed out that the inflammatory processes are per se anabolic.

With respect to inflammation, there are fundamentally 2 types of diets. First is the proinflammatory diet (Westernstyle), which is hypercaloric and high in saturated fat and sugar, refined carbohydrates, fried or processed food, salt, and sweetened beverages. The Western-style diet, which is an animal-based diet, favors anabolism and leads to postprandial inflammation [15], gut microbiota dysbiosis, low-grade inflammatory status, and chronic inflammatory diseases [17-19]. Second is the anti-inflammatory vegetarian/vegan diet, which is basically hypocaloric or regular, favors the catabolism, and downregulates inflammation.

\section{Dietary Habits Influence the Composition of Gut Microbiota}

In humans, the process of digestion and absorption of food occurs in the gastrointestinal tract. At the interface between the gut wall and food an enormous number of microbes, mainly bacteria, called gut microbiota, attend the banquet. It is now known that they have not only a strong influence on food 
absorption, but also on neuroendocrine and inflammatory processes, as well as on brain health [17-19].

The first question to arise is whether nutrition can really influence the composition of gut microbiota and therefore how it may control the number and diversity of gut bacteria. We will see later that the same question is valid for lifestyle and, in particular, physical exercise: have they an influence on gut microbiota and how?

The explanation for the effect of diet is simple: the type of diet changes the composition of gut microbiota by promoting the growth of one or another bacterial species, depending on which is fed.

However, how to check the changes? Actually, the composition of gut microbiota is very complex and highly variable, both with regard to taxa and between individuals (each individual has his/her own "unique" gut microbiota).

For convenience, we can restrict our attention to the 2 most abundant clusters of gut microbiota: 1) the Bacteroidetes/ Prevotella (Cluster B); and 2) the Firmicutes/ Bacteroidetes/ Clostridiales (Cluster F). The above taxa are very suitable biomarkers of gut microbiota, as they correlate well with diet, lifestyle, and disease.

In fact, most of the Bacteroidetes, which are mainly Gramnegative, love to sit at the table of vegans, as they can use as fuel the resistant starch and oligosaccharides present in fibers (inulin and galacto-oligosaccharides) that are not digested in the small intestine. The bacteria fed with fibers produce in the large intestine short-chain fatty acids (SCFAs), such as acetate, propionate, and, in particular, butyrate [20].

However, most of the Firmicutes, which are mainly Grampositive, prefer to sit at the table of Western diet dishes, which are based on high animal fat and refined carbohydrates, as they do not have the machinery for the digestion of complex carbohydrates and prefer macronutrients of animal origin (saturated fatty acids and proteins). Animal-based diets are often energy-dense and poor in fiber. The bacteria that cannot digest fiber and use fat, protein, and simple sugars for their growth are also the more suited to harvest the energy taken in excess, as in the case of the Western diet [21].

In the study of Tilg et al. [21], it was shown that in the transition from a diet rich in carbohydrates, but low in fat, to a high-carbohydrate/high-fat diet, gut microbiota changes: the number of Bacteroidetes species decreases, while the presence of Firmicutes first increases and then prevails, mainly in the form of Mollicutes, which are parasites. The overall microbial diversity decreases dramatically. Tilg et al. [21] concluded that gut microbiota respond adequately to the change of feeding, selecting bacteria that have a higher metabolic capacity and are more suited to extract energy from a Western-style diet.

In the study by De Filippo et al. [22], fecal samples of African children, whose diet was hypocaloric (662-992 kcal/day) and rich in fiber (10 g/day), were compared with those of Italian children, whose diet was an energy-dense, Western-style diet, based on meat, animal fat, refined carbohydrates, and sweetened beverages (1068-1512 kcal/day) with a limited amount of fiber (5.6 g/day). De Filippo et al. [11] observed 2 different populations of gut microbiota depending on the different diets. Bacteroidetes (B) (mainly Prevotella and Xylani species) prevailed in the fecal samples of African children $(73 \%)$ over the Firmicutes (F) (12\%), whereas in the samples of the Italian children Firmicutes (F) (51\%) prevailed over Bacteroidetes (B) (27\%).

The above studies, and others not mentioned here, demonstrate that dietary habits determine the composition of gut microbiota and change it from a "healthy" to a dysbiotic microbiota and vice versa.

\section{The "Healthy" Gut Microbiota}

In general, the human gut microbiota can quickly adapt to diet changes. This is demonstrated by the fact that we can change our diet without problem, even from day to day. This is because the diversity of gut microbiota populations is very high $[23,24]$.

David et al. [25] showed that diets can rapidly alter the composition of human gut microbiota, but if the diet is not prolonged, the composition of gut microbiota returns to its original values within a couple of days. An effective change of gut microbiota composition requires persistent dietary habits.

Once clarified that the composition of gut microbiota can change according to the type of diet, it remains to be explained what this has to do with health or illness; in other words, what does it mean to have a healthy or a dysbiotic intestine.

Indeed, what is a "healthy" gut microbiota? To assess what a healthy gut microbiota is would mean identifying the microbial ecosystems of healthy individuals, which is not quite possible, owing to the high variability of the population associated with a healthy condition. A specific set of microbes that can be associated with a healthy status in all individuals does not exist, as it depends on geographical locations, dietary habits, lifestyle, genetics, and early-life exposure. Every individual, every population, has its own story. It would be better to correlate a healthy status at a functional level rather than at a taxonomic level.

The premises for a healthy status of the gut are that bacteria are well represented therein in number and remarkable diversity. Diversity ensures a greater capability to resist to stress, infections, or metabolic changes, and to recover afterwards. Thus, is it better to have a healthy microbiota with a high biodiversity or with a high gene count [26]? As reported in the article by Tilg et al. [21], changing the diet into one rich in refined carbohydrates and animal fat leads not only to a prevalence of Firmicutes, but also to "a dramatic fall of overall diversity of gut microbiota". 
If so, we can agree that an energy-dense Western-style diet, based on high animal fat/ high sugar, is unhealthy because it leads to a lower representativeness of different bacteria. This decrease cannot be ascribed only to the fact that vegetarian/vegan-diet bacteria do not receive enough of their preferred food, but also because they have to encounter environmental changes less suitable for their life, owing to the prevalence of "carnivorous" bacteria.

\section{Two Classes of Bacteria: Those Based on an Animal Western-Style Diet (W-diet) and Those Based on a Vegetarian/Vegan Diet (V-diet) — An Arbitrary Classification}

Once we have established that diet can change the composition and biodiversity of gut microbiota, we have to determine what the consequences of such modifications are, including the local environmental changes in the gut, that influence the relationships between gut microbiota and the host.

By addressing this point, we realized that the Firmicutes/ Bacteroidetes $(\mathrm{F} / \mathrm{B})$ ratio is no longer adequate to describe the effects of the Western and vegetarian/vegan diets on gut microbiota. Indeed, owing to their relative abundance, the F/B ratio may be an artifact [27], and may not adequately match the change in intestinal ecology determined by the different diets.

This is easier to understand if we consider that among the vegan-diet bacteria there are both Firmicutes (e.g., Clostridium, Faecalibacterium, Lactobacillus, Eubacterium, and Roseburia, all Gram-positive bacteria), Bacteroidetes (e.g., Prevotella and Bacteroides, both Gram-negative), and Actinobacteria such as Bifidobacterium (Gram-positive), among others. In this context, this heterogeneity explains why the separation into Western-style diet and vegetarian/vegan diet bacteria can be more useful than the $\mathrm{F} / \mathrm{B}$ ratio.

Therefore, in the continuation of this work, we will reduce the gut microbiota population into 2 main arbitrary classes, taking into account only their propensity for one or the other diet. In the first class we have bacteria that prefer the Westernstyle diets (low in fiber); in the second class we have bacteria that prefer the vegetarian/vegan diets and that are capable of digesting resistant starch and complex oligosaccharides.

\section{Characteristic Habitats Correspond to Specific Gut Microbiota Populations}

\section{The World of Bile Acids Produced by Western-Style Diet Bacteria in the Intestine}

The prevalence of either Western-style diet or vegetarian/vegan diet bacteria have specific effects on the intestinal habitat.

With regard to the Western-style diet bacteria, David et al. [25] showed that administration of an "animal-based diet" led to a noteworthy fall in bacterial diversity, with enrichment of Bilophila wadsworthia, Alistipes putredinis, and Bacterodes species, whereas administration of a plant-based diet led to higher bacterial diversity and enrichment of the saccharolytic microbes Roseburia, Eubacterium rectale, and Faecalibacterium prausnitzii. The genus Prevotella was the most sensitive to the intake of fibers.

The decrease of bacterial diversity in the animal-based diet was explained by the fact that high fat intake leads to the production of a high amount of bile acids, such as deoxycholic acid [25] and lithocholic acid [28]. Many bacteria, mainly devoted to a plant-based diet, do not survive in the deoxycholate environment, whereas those on an animal-based diet, such as B. wadsworthia, A. putredininis, and Bacterodes species, are bile-resistant. Incidentally, it is to be noted that the $B$. wadsworthia is a dangerous opportunist linked to inflammatory bowel disease, whereas the presence of $F$. prausnitzi is correlated with a healthy gut microbiota [29].

In conclusion, one important aspect of the Western-style diet bacteria is the production of bile salts, which causes a reduction in microbial diversity. However, in the case of a vegetarian/ vegan diet, the saccharolitic bacteria are fed and are not destroyed, and the diversity of gut microbiota is saved. The same as for the vegetarian diet is true for lifestyle, in particular doing physical activity instead of being sedentary [30].

\section{The Beautiful Butyrate World of Vegetarian/Vegan Diet Bacteria}

As the structure of polysaccharides is very complex, much more than that of DNA and proteins, being made of $>120$ monosaccharides, linked and branched in several ways, the hydrolysis of carbohydrate polymers requires a lot of enzymes. This means that digestion by gut microbiota of such complex carbohydrates drives microbial diversity.

Accordingly, vegetarian/vegan diet (V-diet) bacteria are required in larger numbers than Western-style diet bacteria, because their substrates are very complex and require a very large number of hydrolytic enzymes to degrade dietary fibers to oligosaccharides and monosaccharides, which then go on to fermentation to form the SCFAs acetate, propionate, and butyrate [31].

The colonic vegetarian/vegan diet bacteria are protective and anti-inflammatory, mostly because of their production of SCFAs, in particular butyrate [32]. The most important activities for SCFAs are the following [33]: 1) they induce Foxp $3^{+}$ $\mathrm{T}$ regulatory (Treg) cells and tolerance; 2 ) they induce the secretion of IgA from B cells; 3) they inhibit pathogens, also by "competitive exclusion"; 4) they promote the secretion of mucus by gut epithelial cells; 5) they contribute to the integrity of the intestinal barrier, in particular by stimulating the formation of the proteins of tight junctions, and promoting tissue repair and wound healing; 6) they inhibit the proinflammatory transcription factor nuclear factor kappa B (NF-KB) and reduce oxidative stress. 
Particularly important is butyrate. Butyrate is the main energy source of epithelial cells of the colon and protects them by decreasing the $\mathrm{pH}$. A lower $\mathrm{pH}$ reduces the solubility of bile salts and the absorption of ammonia, and increases mineral absorption.

Other symbiotic products of gut microbiota that are useful for the host are mentioned in Fig. 1: polysaccharide A [34], which stimulates immunologic development of the host, and the microbial anti-inflammatory molecule (MAM), an inhibitor of NF-kB produced by $F$. prausnitzii, which is deficient in Crohn's disease [35].

Moreover, brain disorders may be associated with imbalances in protein acetylation and may show transcriptional dysfunction. Histone acetylation is altered in numerous stressrelated disease states. Histone deacetylase inhibitors represent a promising therapeutic option to correct these deficiencies and butyrate is one of the most effective histone deacetylase inhibitors [36].

Finally, dietary tryptophan is metabolized by gut microbiota into agonists of the aryl hydrocarbon receptor (AHR). These metabolites activate AHR signaling in astrocytes and suppress or reduce central nervous system inflammation. It is important to note that in persons with MS the circulating levels of AHR agonists are lower [37].

\section{Intestinal Endotoxemia and Failure of Intestinal Barrier Integrity}

With the animal diet, Western-style diet bacteria grow and prevail on the vegetarian/vegan diet bacteria, which miss their vegetarian/vegan diet and do not survive in the presence of bile acids. With the decrease of vegetarian/vegan bacteria their symbiotic action is lost. In particular, their effect on the compaction of desmosomes [38] and tight junctions is reduced. A high-fat diet induces the production of interleukin (IL)-17A. IL-17A decreases the expression of peroxisome proliferatoractivated receptor- $\alpha$, which is anti-inflammatory and inhibits fatty-acid oxidation [39]. The increase of the endotoxin lipopolysaccharide (LPS), resulting from the changes of gut microbiota composition caused by the Western-style diet, activates Toll-like receptor (TLR) 4 receptors, stimulates T helper cell (Th)17 differentiation [40], increases phosphorylation of the proinflammatory transcription factor NF- $\mathrm{KB}$ and the release of inflammatory mediators, and induces the opening of the gastrointestinal barrier, allowing the passage of LPS, T cells, cytokines and bacteria, and the rising of systemic inflammation (Fig. 2).

In the study by Pendyala et al. [41], 4 weeks of feeding with a Western-style diet increased the plasma levels of LPS by $71 \%$. In another study, the levels of plasmatic and fecal LPS were found to correspond to an increase in the F/B ratio in aged mice.

In conclusion, a Western-style diet leads to gut dysbiosis, LPS-inducible low-grade inflammation, and chronic inflammation. Obviously, a persistently altered microbiota is associated with a persistent low-grade inflammation.

However, what is the link between diet, gut microbiota, and neuroinflammatory diseases?

\section{Gut Dysbiosis and Low-Grade Inflammation as Triggers of Neuroinflammation}

It is more or less acknowledged that gastrointestinal diseases, such as inflammatory bowel disease and colonic cancer, are
Fig. 1 A schematic representation of the effects of diet on gut microbiota. Gut is represented as the labyrinth at the Cathedral of Chartres, in France SCFA = short-chain fatty acid; AHR $=$ ArylHydrocarbon receptor; Treg = regulatory $\mathrm{T}$ cell; $\mathrm{Th}$ $=\mathrm{T}$ helper; $\mathrm{LPS}=$ lipopolysaccharide; $\mathrm{TNF}=$ tumor necrosis factor; $\mathrm{IL}=$ interleukin; $\mathrm{BBB}=$ blood-brain barrier; VDR = vitamin D receptor

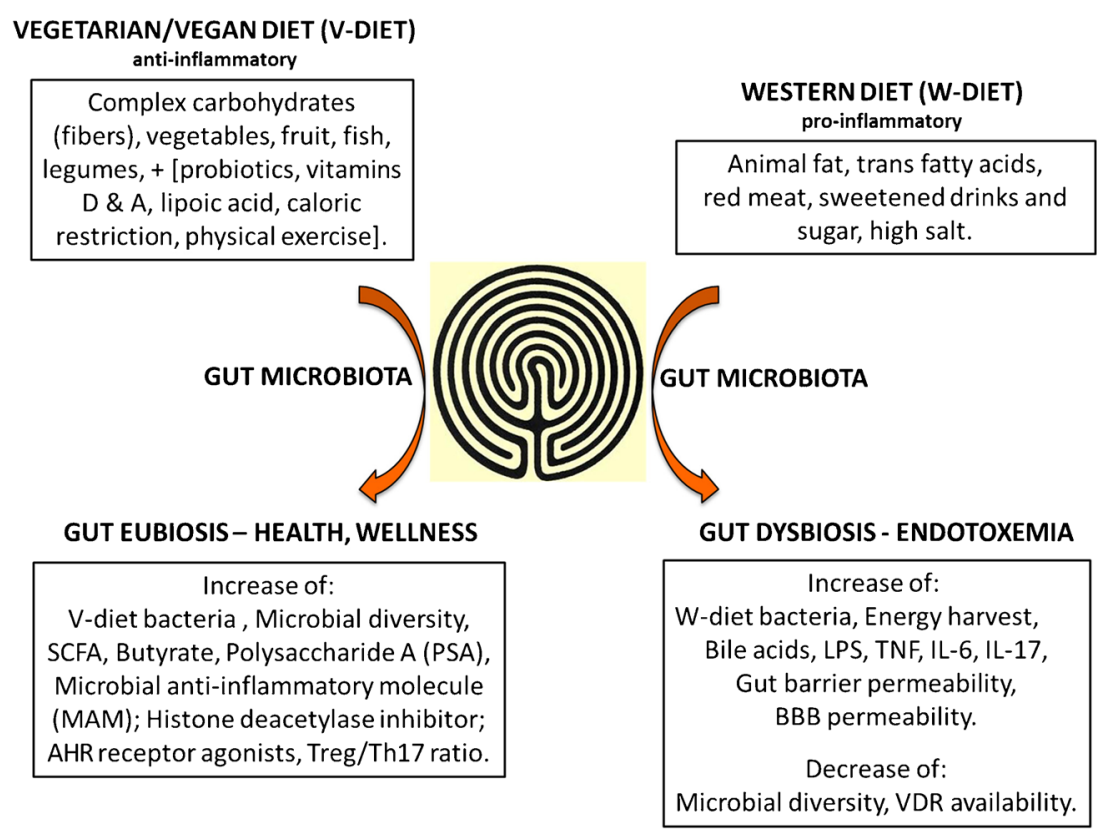


Fig. 2 The pathway from diet to neuroinflammation through gut dysbiosis Treg $=$ regulatory T cell; $\mathrm{Th}=\mathrm{T}$ helper; $\mathrm{LPS}=$ lipopolysaccharide; $\mathrm{BBB}=$ blood-brain barrier
WESTERN DIETS \& LIFE STYLE

Animal fat, red meat, fried food, sweetened drinks and sugar, high salt, low in fiber, alcohol, sedentary lifestyle, processed food

DYSBIOTIC GUT MICROBIOTA

[high ratio W-diet bacteria / V-diet bacteria; low biodiversity]

INTESTINAL INFLAMMATION

- Inflammation, and alteration of intestinal immunity;

- Low Treg/Th17 ratio in the intestinal mucosa;

- Increase of LPS. Disruption of the intestinal barrier

SYSTEMICINFLAMMATION

- Translocation of LPS, peptides, proteins, activated T-cells, gut bacteria into circulation.

- Low-grade endotoxemia (>200pg/ml LPS)

NEUROINFLAMMATION

- Increase of BBB permeability. Passage of pro-inflammatory molecules and cells. Activation of microglia and astrocytes. Damage to myelin and/or neurons. associated with a dysbiotic microbiota. Alteration of gut microbiota has also been observed with obesity, diabetes, allergy, arthritis, and cardiovascular diseases. More recently, it has been suggested that gut microbiota might have a role in neurological disorders $[42,43]$. However, we are just at the beginning of the story, which, at present, is only at a descriptive level, and it is not yet clear what the role of gut microbiota in neuroinflammation and neurodegeneration is.

In 2011, we suggested that gut dysbiosis might influence the course of MS [11]. This suggestion was substantiated by the work of Berer et al. [44], which showed that gut microbiota is required for the development of experimental autoimmune encephalomyelitis (EAE) induced with the autoantigen myelin oligodendrocyte glycoprotein (MOG), as germ-free mice did not developed any disease.

The idea that the gut microbiota has a direct role in neurological disorders has been reinforced by other observations. People with MS have a distinct gut microbiota [45-47] and individuals with Parkinson's disease (PD) also have specific fecal gut microbes compared with healthy controls [48].

The question arises of whether gut dysbiosis is associated with an infectious burden, as suggested for PD and Alzheimer's disease (AD) $[49,50]$, or if it is effective in itself. To understand this point, we show in Fig. 2 a possible path from a Western diet through to neuroinflammatory diseases via the change of gut microbiota composition.

As shown in Fig. 2, proinflammatory dietary habits change first of all the composition of gut microbiota: there is an increase of Western-style diet bacteria, which prevail over saccharolitic bacteria, and a reduced bacterial diversity in the gut. This change leads to an increase in LPS; an increase in the Th17/Treg ratio; expression of proinflammatory cytokines, such as IL-6, IL-1 $\beta$ and tumor necrosis factor- $\alpha$; onset of intestinal inflammation, downregulation of occludin, the tight junction protein; and increase of intestinal barrier permeability. Indeed, the gut microbiota has a strong influence on gut barrier integrity and the first consequence of dysbiosis is the increase of gut barrier permeability. An altered permeability of the intestinal barrier has been recently reported in relapsingremitting MS [51].

The disruption of the intestinal barrier allows the passage of LPS, peptides, and proteins of immunological interest, and also the slow translocation in the circulation of gutassociated lymphoid tissue (GALT)-activated autoreactive lymphocytes [52] and of gut bacteria [47]. Indeed, people with MS showed a slow translocation of bacteria from gut into the circulation [47]. It is not yet known if the translocating gut bacteria have a specific role in the development of MS, and if this will allow microbiota therapies for patients in MS in the future.

The low-grade endotoxemia (LPS > $200 \mathrm{pmol} / \mathrm{ml}$ ) sustains a low-grade systemic inflammatory status and the progression of MS. Besides MS, other chronic neuroinflammatory disorders, such as PD and AD, stroke, and psychiatric disorders may be involved.

For this to happen, the blood-brain barrier (BBB) must lose its integrity. This is the premise for neuroinflammatory processes. The disruption of the BBB may be ascribed not only to infectious agents, trauma, fever, and other triggering agents, but may also simply be due to those molecules and cells contributing to the low-grade inflammation originating from gut dysbiosis. In fact, it may be expected that the same factors that have led to a persistent leakage of the intestinal barrier in the course of intestinal inflammation are also able to cause BBB disruption. 
In conclusion, the main link between nutrition and neuroinflammatory disorders is gut dysbiosis, caused by wrong dietary habits. Gut dysbiosis causes low-grade endotoxemia, disruption of the gut barrier, leakage of LPS, protein, and cells of immunological importance into the circulation, and then the rising of low-grade systemic inflammation, breakdown of the BBB, activation of microglia and astrocytes, and, finally, chronic neuroinflammatory disorders.

\section{The Role of Diet in MS and Other Neuroinflammatory Diseases}

Up to 7 years ago diet was by no means considered to have an influence on the course of MS, and the role of the gut microbiota in MS was judged to be only speculative, without any foundation. The impact of food on the intestine was taken in consideration only in case of intestinal disorders, obesity, cardiovascular disease, and cancer. It is now clear that our dietary habits influence our health, including neurological disorders, such as MS, PD, AD, autism, stroke, mood, and irritability.

In light of what has been said so far, a healthy diet must have a high fiber content (> 10-15 g/day) and must be varied as much as possible, as this can allow a larger number of different microbial species.
With regard to low-grade inflammation and its control by a dietary intervention, we must be aware that food contains factors that have pro- or anti-inflammatory properties. In the case of chronic inflammatory diseases, the diet should be antiinflammatory and therefore be mainly vegetarian. In the following subsections we will only briefly mention pro- and antiinflammatory foods and their properties, as this has been covered previously [10-13].

\section{Proinflammatory Dietary Factors}

A list of the proinflammatory dietary factors is provided in Table 1.

Saturated fatty acids (SFAs), the major components of animal fat, were first taken into consideration by Swank in 1950 [53] and their negative effects on the course of MS were reported by Swank and Goodwin [54] much later. The adverse effects of SFAs were ascribed to their proinflammatory character. SFAs are, indeed, very similar to LPS, as they are structural components of lipid A present in all pathogenic LPS [55]. As with LPS, SFAs bind to the TLR-2 and TLR-4, and activate the pr-inflammatory transcription factor NF- KB $[56$, 57]. Their persistent intake leads to a dysbiotic gut microbiota and to low-grade endotoxemia.

Table 1 Proinflammatory dietary factors

Food components Effects

1) Saturated fatty acids [prevalently of animal origin (butter, whole milk, cheese, meat, sausages); coconut oil and palm oil]

a) Binding to TLR-2 and TLR-4 and activation of the proinflammatory transcription factor, NF- $\mathrm{kB}$

b) Change of gut microbiota composition; gut dysbiosis

c) $\uparrow$ IL-17A, $\downarrow$ PPAR- $\alpha$, and $\beta$-fatty acid oxidation

2) Trans fatty acids (in margarine, meat, snacks, French fries, and other fried food) a) Interference with natural "cis" unsaturated fatty acids

b) Intestinal inflammation; upregulation of Th17 cells

c) $\uparrow$ Cholesterol; $\uparrow$ abdominal fat; $\uparrow$ weight gain

3) Red meat

a) Iron is nitrosylated to nitroso compounds

b) Abnormal iron deposits in MS

c) GGT and hsCRP increase

d) Heterocyclic amines during cooking

e) Anti-Neu5Gc antibodies

f) Arachidonic acid (eicosanoids)

g) Activates the Th17 pathway

4) Sugar, refined cereals, sugar-sweetened beverages and low-fiber intake

a) Insulin increase - high calories

b) Postprandial inflammation

c) Biosynthetic pathways

d) Feeding specific gut microbiota leading to dysbiosis

5) Increased dietary salt intake

a) Induce pathogenic Th17 cells and proinflammatory cytokines in EAE

6) Proteins of the MFGM: BTN, a major protein of the MFGM, is very similar to MOG, the MS candidate autoantigen.

a) MOG/BTN cross-reactive antibodies have been found in MS, in autism and in CHD

b) BTN, as MOG, can induce EAE

MFGM = milk fat globule membrane; $\mathrm{BTN}=$ butyrophilin; $\mathrm{MOG}=$ myelin oligodendrocyte glycoprotein; $\mathrm{MS}=$ multiple sclerosis; TLR = Toll-like receptor; NF-KB = nuclear factor kappa $\mathrm{B}$; IL= inteleukin; PPAR = peroxisome proliferator-activated receptor; Th = T helper; GGT = gamma-glutamyl transpeptidase; hsCRP $=$ high-sensitivity C-reactive protein; Neu5Gc $=N$-glycolylneuraminic acid; EAE = experimental autoimmune encephalomyelitis; $\mathrm{CHD}=$ coronary heart disease 
Trans fatty acids are vegetable oils that have been hydrogenated. They interfere with the metabolism of natural unsaturated fatty acids, which have the cis configuration. The intake of trans fatty acids is associated with gut inflammation and upregulation of Th17 cells.

Red meat contains more heminic iron than white meat. In processed, nitrite-preserved red meat the formation of nitroso compounds may be high. Moreover, red meat and milk products have $\mathrm{N}$-glycolylneuraminic acid (Neu5Gc), a major sialic acid, which we are no longer able to express. Dietary Neu5Gc can increase chronic inflammation, as we have circulating anti-Neu5Gc antibodies. Finally, meat contains arachidonic acid [the omega-6 (n-6) polyunsaturated fatty acid (PUFA), which is the precursor of proinflammatory eicosanoids (prostaglandins, thromboxanes, leukotrienes)], which activates the Th17 pathway.

In Western diets, the high intake of sugar-sweetened beverages and refined cereals, with low fiber content, leads to the increase of insulin production, which, in turn, upregulates the biosynthesis and production of arachidonic acid.

High dietary salt intake can induce pathogenic Th17 cells and related proinflammatory cytokines [58].

Milk fat is dispersed in vesicles, each of which is protected by a membrane with particular proteins called "proteins of the milk fat globule membrane" (MFGM) [59]. These minor milk proteins (only about $1 \%$ of the total) have an informational rather than a nutritional value, as they are needed for correct formation of the digestive, nervous, and immune systems in infants. Obviously, such information is no longer required in adulthood. The MFGM proteins may have a relevant role in MS. In fact, the major MFGM protein (40\% of the total), butyrophilin, is very similar to MOG, one of the candidate autoantigens in MS. Both butyrophilin and MOG induce EAE and share cross-reactive antibodies in MS, autism, and coronary heart disease [59]. On these grounds, persons with MS should drink skimmed milk.

\section{Proinflammatory Dietary Habits: Hypercaloric Diets and Postprandial Inflammation}

After an energy-dense meal, a temporary low-grade inflammatory response may follow, the extent of which depends on the quantity and type of food [15]: 1) calorie intake - the higher the calories, the more the oxidative stress is induced; 2) glycemic load of the meal-acute postprandial glycemic peaks may induce a release of insulin much higher than necessary; 3) lipid pattern - saturated animal fat, trans fatty acids, and omega-6 (n-6) long-chain PUFAs all promote postprandial inflammation. Postprandial inflammation is reduced by $n-3$ PUFAs, polyphenols, caloric restriction, and physical exercise (mentioned below), which are anti-inflammatory.
Table 2 Anti-inflammatory dietary molecules and microorganisms

Group 1 (recognized as intrinsic to the body)

1.1. Omega-3 (n-3) polyunsaturated fatty acids (fish oil)

1.2. Vitamins (D, A, $\left.B_{12}, \mathrm{C}, \mathrm{E}, \mathrm{PP}\right)$

1.3. Carotenoids [lycopene (tomato): retinoic acid, activates the anti-inflammatory RXR receptor

1.4. Oligoelements (selenium, zinc, magnesium)

1.5. Thiolic compounds (lipoic acid, $N$-acetyl-cysteine)

Group 2 (recognized as foreign to the body)

2.1. Polyphenols [flavonoids (quercetin, catechins...), nonflavonoids (resveratrol, curcumin, hydroxytyrosol...)]

Group 3 (improve the intestinal microbial balance)

3.1. Probiotics (e.g., Lactococcus lactis, Bifidobacterium lactis, Clostridium butyricum...)

Group 4 (nutrients for symbiotic gut microbiota, reduce inflammation and give health benefits to the host)

4.1. Prebiotics (including inulin, bran, lactosucrose, and oligofructose)

$\mathrm{RXR}=$ retinoid $\mathrm{X}$ receptor

\section{Anti-Inflammatory Dietary Molecules and Microorganisms}

Specific dietary molecules are able to downregulate inflammatory processes by inhibiting proinflammatory transcription factors as NF- $\mathrm{KB}$ and activator protein 1 , or by modulating the activity of enzymes involved in inflammatory events.

The most important anti-inflammatory dietary compounds are listed in Table 2. Most of them, but not the n-3 PUFAs, are antioxidants, which make them very useful, as oxidative stress is an important component of the inflammatory processes leading to degradation of myelin and axonal damage. Oxidative stress and inflammation are present in relapsing-remitting and in primary and secondary progressive MS $[60,61]$.

As reported in Table 2, the anti-inflammatory molecules can be divided into 2 groups.

\section{Group 1}

The compounds of the first group can be considered "intrinsic" to the body or, in other words, familiar with the metabolome. They usually participate in our metabolism, can be either synthesized or stored in our body, or may act as coenzymes or cofactors in catalysis. They are: 1) omega-3 (n-3) PUFAs (fish oil); 2) vitamins D, A, B ${ }_{12}, \mathrm{C}, \mathrm{E}$, and PP; 3) carotenoids (lycopene); 4) oligoelements (selenium, zinc, magnesium); 5) thiolic compounds (lipoic acid; $N$-acetilcysteine).

Omega-3 Polyunsaturated Fatty Acids n-3 essential fatty acids (EFAs) and PUFAs are important fatty acids required to balance the proinflammatory activity of SFAs, n-6 PUFAs, and trans fatty acids [13, 62, 63]. 
The EFAs, linoleic acid (n-6) and linolenic acid (n-3), are found in vegetable and vegetable oils. Linoleic acid is the precursor of arachidonic acid and its proinflammatory eicosanoids, whereas linolenic acid is the precursor of n-3 PUFAs.

With regard to PUFAs, it is important to note that $n-6$ (proinflammatory) and n-3 (anti-inflammatory) fatty acids have opposite effects and their presence in the diet should be equivalent [63]. However, in Western diets the ratio of $n-6 / n-3$ is increased from 6 to 15 times, or even more, which leads to a higher incidence of chronic inflammatory diseases. The n-3 long-chain PUFAs, eicosapentaenoic acid and docosahexaenoic acid, may derive from n-3 linolenic acid, or from the intake of seafood and fish oil. They inhibit the synthesis of proinflammatory eicosanoids, fatty acids, and cholesterol, and stimulate the oxidation of fatty acids. On this basis, in chronic inflammatory diseases such as MS, n-3 EFAs and n-3 PUFAs should prevail in the diet over the $\mathrm{n}-6$ fatty acids.

Vitamins Vitamins, such as vitamin D [9], vitamin A [64, 65], vitamin E [66], vitamin B12 [67], and niacin [68], but, in particular, vitamins $\mathrm{D}$ and $\mathrm{A}$, have fundamental antiinflammatory and metabolic roles.

Carotenoids Among the carotenoids, the most important is lycopene (tomato, water melon, pink grapefruit) [69]. To be a very strong antioxidant, lycopene can form $\beta$-carotene and retinoic acid, the latter of which can activate the retinoid $\mathrm{X}$ receptor (RXR).

Oligoelements Oligoelements, such as selenium [70], zinc [71], and magnesium [72], have important immunomodulatory, antioxidant, and anti-inflammatory properties. Zinc, which is present in myelin and binds to myelin basic protein, is also important for myelin assembly [73].

Thiolic Compounds Compounds containing thiol groups ($\mathrm{SH})$, such as $\alpha$-lipoic acid, glutathione, and $N$-acetylcysteine, should be taken into consideration as dietary supplements to be used for the complementary treatment of MS. ALA is antiinflammatory and stabilizes the integrity of the BBB $[74,75]$. NAC might also be useful in neurological disorders, as it passes through the BBB and protects against inflammation [76].

\section{Group 2}

The second group of anti-inflammatory dietary factors is made of natural substances that can be considered to be "foreign" to our body. The most important of this group are the polyphenols [13]. They can be metabolized, but not synthesized or stored in our organism.

On the basis of their structure, polyphenols are recognized as flavonoids (quercetin and cathechins) and nonflavonoids (resveratrol, curcumin, and hydroxytyrosol). They are present in vegetables, fruits, cereals, legumes, spices, herbs, chocolate, wine, tea, and coffee. Their solubility and bioavailability are very poor [77]. They show anti-inflammatory, antiangiogenic, and antiviral properties, and upregulate the catabolism [13, 78]. An important aspect of polyphenols is that they can inhibit some cytochrome P450 enzymes (CYPs) and therefore they may interfere with drugs [79]. It has been found that the anti-inflammatory effect of polyphenols in vitro may depend on their chemical structure [80]; thus, a mixture of flavonoids and nonflavonoids may be more effective than supplementation with only 1 type of polyphenols [81].

Microorganisms, called probiotics, such as Lactococcus lactis, Bifidobacterium lactis and Clostridium butyricum, can improve the intestinal microbial balance [82]. Their administration in diseases related to gut dysbiosis is fundamental.

Prebiotics, such as inulin, bran, lactosucrose, and oligofructose, are preferential nutrients for colonic bacteria. As already mentioned, dietary fibers are made of soluble or insoluble complex carbohydrates that cannot be digested by our body and therefore do not influence our metabolism but represent an important food source for symbiotic gut microbes, which can give health benefits to the host. Dietary fibers are anti-inflammatory and compete with dysbiotic gut microbiota. Their intake must be $>10 \mathrm{~g} /$ day and must be as varied as possible to increase the diversity of gut microbiota.

\section{The Impact on Gut Microbiota of Chemicals Added to Processed Food}

As mentioned above, food does not cause damage to the host per se, given that it is natural, not processed, not adulterated, and well digested. However, processed foods often contain additives, artificial flavorings, preservatives, emulsifiers, antibiotics, or contaminants, such as organic pollutants, heavy metals, pesticides, and herbicides. These minor ingredients of food may have deleterious effects on gut microbiota [83, 84].

Dietary emulsifiers, such as carboxymethylcellulose and polysorbate 80 , may lead to a decrease of gut microbiota diversity, intestinal inflammation, and reduction in mucus layer thickness. Other chemicals that may be present in food are the non-nutrient sweeteners, such as saccharin, aspartame, and stevia: they may have a bacteriostatic effect and change the composition of the gut microbiota. Organic pollutants may have dramatic effects on gut microbiota and organophosphate insecticide has been shown to induce dysbiosis.

Antibiotics are often present in foods and surface waters, although at low concentrations. They are used in livestock either as growth promoters or for prophylactic use. The persistent consumption of food containing antibiotics alters the microbiota composition in the adult, 
leads to a significant decrease of microbial diversity, and causes resistance to antibiotics.

Finally, the intake of rancid foods should be avoided, because they may contain harmful microbes [85].

\section{Proinflammatory Lifestyles}

\section{Smoking}

The role of cigarette smoke in MS is still controversial. Weiland et al. [86] and Munger et al. [4] have found no association between cigarette smoke and relapse rate or disease activity of MS. However, persistent cigarette smoke induces changes in gut microbiota and inflammation in the murine gut [87] and may worsen the course of MS. At the molecular level, cigarette smoke inhibits the sirtuins, deacetylating enzymes that upregulate catabolism and downregulate anabolism and inflammation [13].

\section{Alcohol Consumption}

It seems that alcohol consumption is not associated with MS risk [88]. However, alcohol inhibits the enzyme sirtuin 1 (SIRT1) and activates the transcriptional activity of steroid regulatory element-binding protein $1 \mathrm{c}$, thus promoting the biosynthesis of lipids and inflammation at the expense of oxidative metabolism [13]. Furthermore, the metabolism of ethanol converts nicotinamide adenine dinucleotide molecules to reduced nicotinamide adenine dinucleotide and limits the availability of nicotinamide adenine dinucleotide required for the activity of sirtuins and catabolic pathways, such as glycolysis. Besides, ethanol is a substrate of the P450 enzymes and can interfere with the metabolism of drugs, which are transformed by the same enzymes. Altogether, alcohol is not a nutrient: it interferes with normal metabolism and facilitates the inflammatory process. Finally, alcohol induces alterations in the intestinal immune response as a consequence of the induced dysbiosis and increases host susceptibility to infections [89].

\section{Anti-Inflammatory Lifestyles}

\section{Caloric Restriction (Reduction of Food Intake or Controlled Fasting)}

Caloric restriction, also in terms of intermittent fasting, is a very effective tool to promote a healthy state in living organisms and increase their lifespan and their health span. We are now beginning to understand that the effects of caloric restriction, due a reduction of dietary intake of $20-40 \%$, cannot simply be ascribed to a reduced number of calories.
Caloric restriction upregulates the level of SIRT1; increases the level of adenosine monophosphate and upregulates 5, adenosine monophosphate-activated protein kinase (AMPK) and protein kinase $\mathrm{B}$; increases adiponectin levels and upregulates or activates its receptors, downregulating insulin, oxidative damage, lymphocyte activation, and the progression of experimental models of multiple sclerosis [90]. The effects of caloric restriction are mimicked by agonists as resveratrol and other polyphenols, acting on the same targets (SIRT1, AMPK).

Among the effects of fasting are the decrease of insulin and the increase of glucagon. Insulin stimulates the activity of the enzymes delta- 5 and delta- 6 desaturases, which lead to the inflammatory $n-6$ PUFA arachidonic acid, whereas glucagon inhibits the above enzymes and the production of arachidonic acid.

In their study, Choi et al. [91] showed that a fasting mimicking diet for 3 days, followed by a Mediterranean diet for 6 months, was effective in ameliorating murine EAE. Accordingly, the Treg/Th17 ratio increased, whereas cytokine levels decreased.

Together with an anti-inflammatory diet, and the administration of dietary supplements (in particular vitamins D and A), caloric restriction is one of the better tools with which to ameliorate the symptoms of MS.

\section{Physical Exercise}

Physical exercise is now a common practice applied to decrease the symptoms of chronic fatigue and prevent or slow the onset of disability in patients with MS [92]. It should be considered in a holistic context in which diet, exercise, therapy, and social communication contribute to the wellness of those with MS [93].

Physical exercise is also effective at the molecular level, as it exerts its effects on the AMPK/sirtuins/peroxisome proliferator-activated receptor network, and upregulates oxidative metabolism, while downregulating biosynthesis and inflammation [13]. Moreover, physical exercise ameliorates quality of life, stimulates the production of antiinflammatory cytokines, decreases the levels of leptin, and increases adiponectin levels and adiponectin receptor activity [94, 95].

Studies performed on rats showed that physical exercise could alter the composition and diversity of gut bacteria $[96,97]$.

\section{Alternative or Complementary Dietary Interventions in MS}

There have only been a few clinical studies on the impact of diet on the course of MS and most refer to treatments with 
single dietary supplements. The efficacy of dietary interventions or administration of dietary supplements in the course of MS has not yet been studied [98].

It should be clear that diet alone or administration of a single dietary supplement can be suitable for prevention and to promote wellness, rather than for cure. Nutritional intervention is not a drug and needs months to influence the course of the disease.

However, there is a strong demand for dietary guidelines and a number of diets have been developed: "Kousmine" [99]; "Swank" [100]; "Paleolithic" [101]; "Mediterranean" [102]; "McDougall" [103]; "gluten-free" [104]; "Longo diet, mimicking fasting" [91]; "anti-inflammatory" [105]. With exception of the Kousmine [99] and Swank [100] diets, all of the abovementioned diets are recent, a sign of the growing importance of diet in chronic inflammatory diseases, cancer, and senescence.

Our approach is to consider a diet from the point of view of its anti-inflammatory properties. Dietary factors of the antiinflammatory diet activate transcription factors and enzymes that upregulate catabolism and downregulate inflammation, and promote a healthy, well-balanced gut microbiota.

\section{The Anti-Inflammatory Diet}

Recently, we have carried out a pilot study to assess the influence of nutritional intervention on inflammatory status and wellness in people with MS [105]. The anti-inflammatory diet was calorie-restricted, mostly vegetarian, and associated with the administration of supplements such as fish oil, lipoic acid, resveratrol and vitamins, in particular vitamin $\mathrm{D}$ at a low dosage. Zymographic analysis [106] of the activity of serum matrix metalloproteinase-9, or gelatinase B, a marker of inflammatory status, showed its decrease in the majority of patients, demonstrating the effectiveness of dietary intervention [105].

\section{On the Administration of Vitamin D as a Supplement in MS}

Vitamin D is the most promising dietary molecule for the treatment of autoimmune and chronic inflammatory diseases $[14,107]$. The deficiency $(<20 \mathrm{ng} / \mathrm{ml})$ or insufficiency $(21-29$ $\mathrm{ng} / \mathrm{ml}$ ) vitamin $\mathrm{D}[14]$ is generally ascribed to low exposure to sunlight. However, most patients with MS have insufficient levels of vitamin D, independently of their geographical location. For example, MS is also present in sunny Southern Italy [105], as well as in Cyprus [108]. Rather, low levels of active vitamin D might be owing to its altered metabolism or function and not to low exposure to sunlight.

Vitamin D can be supplemented in the form of cholecalciferol. As shown in Fig. 3, the activity of supplemented vitamin $\mathrm{D}$ is independent of sunlight and depends on the activity of

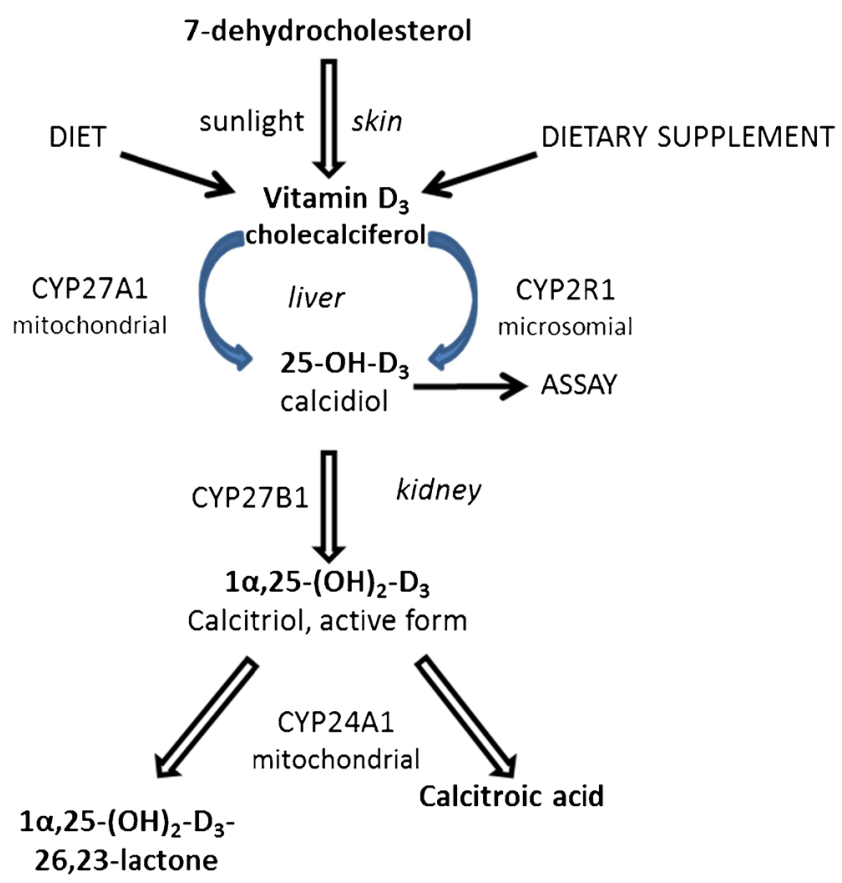

Fig. 3 Cytochrome P450 (CYP) enzymes and the metabolism of vitamin D. Cholecalciferol is formed in the skin from 7-dehydrocolesterol after exposure to sunshine, then hydroxylated in the liver to 25-(OH)D3 (calcidiol) by the P450 enzymes CYP27A1 or CYP2R and subsequently activated in the kidney by CYP27B1 to $1 \alpha, 25-(\mathrm{OH})_{2} \mathrm{D}_{3}$ (calcitriol), the active form of vitamin D. The latter is inactivated by CYP24A1 mainly to $1 \alpha, 24,25-(\mathrm{OH})_{3} \mathrm{D}_{3}$ (calcitroic acid). Therefore, active vitamin D levels depend on the relative rates of its synthesis via CYP27B1 and its modifications via CYP24A1

CYP enzymes. In particular, levels of active vitamin D depend on the relative rates of its synthesis via CYP27B1 and its modifications via CYP24A1. Higher CYP24A1 expression, induced by endogenous compounds or by xenobiotics, might lead to low levels of vitamin D.

\section{The Vitamin D Receptor and the Requirement for Vitamin A}

Another important factor that may contribute to the low levels of vitamin D in MS is the availability of the vitamin D receptor (VDR). As shown in Fig. 4, the anti-inflammatory properties of vitamin $\mathrm{D}$ require the binding to VDR of calcitriol $\left[1,25-(\mathrm{OH})_{2} \mathrm{D}_{3}\right]$. Once it is formed, the complex VDR-D binds to the receptor RXR activated by its ligand retinoic acid (RA), the main metabolite of vitamin A. The heterodimer complex RXR-RA/VDR-D controls the expression of several genes involved in inflammatory and autoimmune processes in chronic diseases by inhibiting the proinflammatory transcription factor NF- $\mathrm{KB}$ and downregulating the synthesis of inflammatory molecules. From here it turns out that the antiinflammatory action of vitamin $\mathrm{D}$ requires the formation of the active heterodimer and therefore the presence of vitamin A. In practice, vitamin D should be supplemented with 
Fig. 4. Role of active vitamin D and vitamin $\mathrm{D}$ receptor (VDR) in inflammation, through their binding to the retinoid $\mathrm{X}$ receptor (RXR), activated by retinoic acid (RA) VDR = vitamin D receptor; $\mathrm{NF}-\mathrm{KB}=$ nuclear transcription factor kappa $\mathrm{B} ; \mathrm{n}-3$ PUFA = omega-3 polyunsaturated fatty acids; $\mathrm{RA}=$ 9-cis-retinoic acid; SIRT- $1 / 2$ = sirtuins (deacetylating enzymes); $\mathrm{NO}=$ nitric oxide; ROS $=$ reactive oxygen species; $\mathrm{MMP}=$ matrix metalloproteinase
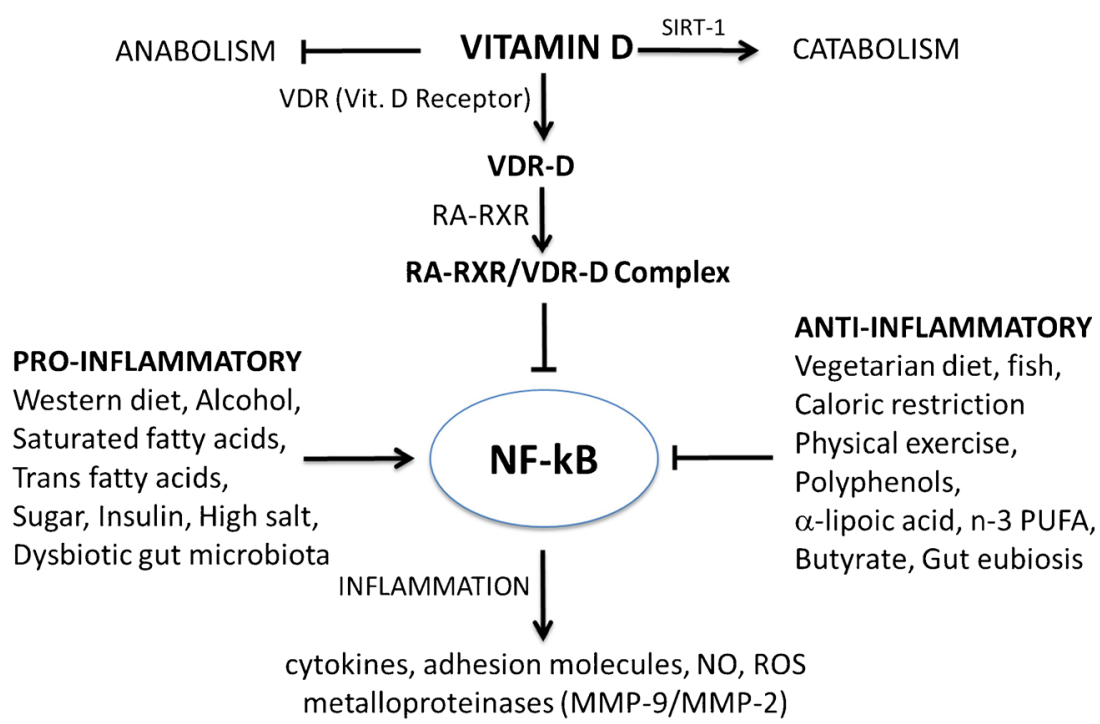

vitamin A. Vitamin A, by itself, has a beneficial role in MS, for its anti-inflammatory and immunomodulatory properties [64], and it has been shown that administration of RA can prevent the development of EAE in Lewis rats [109] and enhances oligodendroglial differentiation and remyelination [65].

The low effectiveness of vitamin D supplementation, if supplemented at low doses, could be ascribed to the eventual polymorphisms affecting the VDR, which has been associated with obesity, inflammation, and alterations of gut permeability [110].

Moreover, the finding that that VDR-D activates SIRT-1 [111] suggests that vitamin D also has an influence on cell metabolism and therefore may have properties similar to those of many other natural dietary supplements, i.e., upregulation of oxidative metabolism and downregulation of inflammation.

\section{Vitamin D and Gut Microbiota}

With regard to the relationship between vitamin D and the gut microbiota, emerging evidence suggests that vitamin D has an important role in maintaining the integrity of the intestinal barrier, as it enhances the tight junctions that control mucosal permeability [112]. Accordingly, vitamin D deficiency leads to disruption of the gut barrier and dysbiosis. Indeed, it has been found that VDR, which is highly expressed in small intestine and colon, regulates the composition of the gut microbiota and its deficiency causes dysbiosis [113]. Lack of VDR in a VDR knockout mice model was found to be associated with gut dysbiosis, and Sun [114] showed that decreased expression of intestinal epithelial VDR leads to fewer butyrate-producing bacteria. As mentioned above, butyrate prevents mucosal inflammation. In addition, Sun [114] found that administration of the bacterial product butyrate increased intestinal VDR expression. Besides its effects on intestinal barrier integrity, VDR has a strong influence on innate immunity and host defense in the gut, and has protective effects on colorectal cancer [115].

\section{Western-style Diet Bacteria, Bile Acids, and Vitamin D}

Our dietary habits can increase or decrease the effectiveness of vitamin D in the gut. In case of Western diet, the Western-style diet gut bacteria contribute to bile acid homeostasis in the host through deconjugation and dehydroxylation reactions to form secondary bile acids. Bile acids as deoxycholic acid and lithocolic acid have at least 2 drawbacks: 1) they create an unsuitable habitat for vegetarian/vegan diet bacteria and reduce biodiversity in the gut; 2) they are ligands for VDR, reduce vitamin $\mathrm{D}$ effectiveness, and increase the risk of diseases.

\section{Administration of Vitamin D and Other Dietary Supplements}

Administration of anti-inflammatory dietary supplements, in particular those of the first group in Table 2, in association with an anti-inflammatory diet, represents a good choice to improve wellness and quality of life in persons with MS, reducing fatigue and delaying the progression of disability. Treatment with dietary supplements should comprise probiotics, prebiotics, vitamin D and vitamin A.

Vitamin D should be supplemented until its serum concentration is $30-60 \mathrm{ng} / \mathrm{ml}$, required for binding to VDR. The dosage of vitamin D administration is still controversial. In our experience, low dosage (about $700 \mathrm{IU} /$ day) is ineffective [105] and higher amounts (> 3500-5000 IU/ day) are required. Other useful dietary supplements are lipoic acid and omega-3 PUFAs (fish oil). A formula with a mixture of n-3 and n-6 PUFAs gave good results [116], 
but in the case of MS we consider a better choice the consumption of $\mathrm{n}-3$ fatty acids only.

Probiotics and prebiotics should be taken often, if necessary. Physical activity should be practiced frequently.

\section{Fecal Microbiota Transplantation}

Fecal microbiota transplantation (FMT), applied for the first time in 1978 to treat Clostridium difficile infection, is a technique based on the transfer of fecal content from a healthy donor to an individual with gut dysbiosis to restore a balanced microbial community. The FMT intervention has proven to be very effective, improving specific chronic diseases associated with gut dysbiosis [117].

At present, FMT is considered to be like an organ transplantation but without the problem of immunological rejection. FMT could be an important therapeutic practice to improve the course of MS, as it has been shown that people with MS have a dysbiotic gut microbiota, and could also be effective in other neurological disorders.

That feces transplants from healthy to sick individuals may be beneficial is substantiated by the observation that older fish live longer after eating microbes released from the feces of younger fish [118, 119]. However, it should be considered that fecal transplants are not well defined in composition. The nature of feces is very composite: in addition to viable bacteria, viruses, archaea, fungi, toxins, and other metabolites are also delivered and contribute to modifications of host gut microbiota [120]. The impression is that this technique is still rather rough and depends a lot on the choice of the donor. Until it is improved, FMT could be avoided if effective intervention is achieved by high-doses of probiotics and prebiotics.

\section{Conclusions}

This work concerns the role of nutrition and dietary supplementation in MS and takes into account the effects they have on the disease through the gut microbiota of the host. In fact, while it is still unclear what nutrition has to do with a chronic neuroinflammatory disease such as MS, it seems now established that the gut microbiota has important effects on the human health. Hence, it is important to investigate its involvement when applying a nutritional intervention with the aim of ameliorating the wellness of persons with MS.

Here, we argue that the link between nutrition, gut microbiota, and MS is low-grade inflammation and that the extent of low-grade inflammation largely depends on dietary habits and their effects on the gut microbiota and the host.

However, the gut microbiota is very complex and dynamic, and its relationships with the host are complex and intricate, as they include not only the relationship between microbe and host (related to how the microbiota affects human health), but also the relationships between host and microbe, and between microbes. This implies both a number of mutual interactions of the microbiota with the host and between neighboring microbes, and a number of related effects-some beneficial, some harmful - on the host microbiome as an ecosystem. Moreover, the relationship between the host and the gut microbiota evolves with different pressures because the microbiota compete within the host ecosystem, whereas the host evolves while keeping the ecosystem under control [121].

What is in common in this complex network is nutrition. Both gut microbiota and the host feed and change accordingly, but it is the host who decides how to feed. Here we describe how persistent dietary habits and vitamins D and A shape gut microbiota composition and influence low-grade inflammation and the course of MS.

To break down the complexity of the topic covered by this study, we have reduced the gut microbiota to two main classes of bacteria (the Western-style diet bacteria and the vegetarian/ vegan diet bacteria) on the basis of their dietary preferences towards 2 main types of diet made available by the host: the Western (W) and the vegetarian (V) diets. The Western-style diet is hypercaloric and proinflammatory, based on animal food and low in fiber; the vegetarian/vegan diet is antiinflammatory and rich in fiber.

This subdivision of gut microbiota has been made on the basis of the food preferred by specific gut bacteria and on the observation that dietary habits change the population of gut microbiota and modify their mutualistic relationship with the host. As mentioned above, the gut microbiota is in a complex symbiotic relationship with the host, as it modulates gut homeostasis, cooperates in the digestion and absorption of nutrients, and interacts strongly with the host at the metabolic, endocrine, immunological, and neurological levels [122].

As shown in this work, the Western-style diet leads to gut dysbiosis and changes the interaction of gut microbiota with the host metabolism and immune system towards a state of low-grade inflammation, whereas vegetarian/vegan diet bacteria provide several molecules useful for the host (mainly SCFAs obtained by the fermentation of fibers, complex carbohydrates indigestible by the host). Other beneficial roles of vegetarian/vegan diet bacteria include their contribution to the integrity of the intestinal barrier, the prevention of colonization by pathogens, and modulation of the development and the activity of GALT. These effects may be lost when the Western-style diet bacteria prevail.

We have shown here that the gut microbiota can adapt very easily to dietary changes; however, only persistent dietary habits cause a persistent change of gut population. This means that the occasional intake of proinflammatory foods is not deleterious. What is deleterious is their prolonged intake. In fact, only in the long run can a Western-style diet establish the conditions (decrease in both vegetarian/vegan diet bacteria 
and gut microbiota diversity) favoring the development of intestinal endotoxemia, an increase of gut barrier permeability, and the onset of low-grade systemic inflammation. Neuroinflammatory disorders can then follow if there is disruption of the $\mathrm{BBB}$, upregulation of microglia and astrocytes, and, eventually, autoimmune processes as in MS, stroke, autism, AD, and PD.

An important question remains: does gut dysbiosis precede MS, or does MS lead to gut dysbiosis [123]? This is the classic "chicken or egg" dilemma.

The question is malicious, as communication along the brain-gut axis is, in fact, bidirectional. What is usually left out in this connection between gut and brain is the nutritional aspect. In fact, what drives the metabolic evolution of host and microbiota ecosystems are dietary habits and lifestyle. These can affect the status of gut microbiota, and set up the premises for disease or for the recovery of a healthy condition, leading to worsening or improvements. It has been shown that people with MS have distinct gut microbiota [45-47]. The question is whether the gut microbiota associated with MS is a result of the disease, or the premise for the low-grade inflammatory status originating from the intestine. Actually, the relationship between BMI and MS indicates that individuals with a high BMI before the age 20 years have a $2 \times$ increased risk [15]. In the 2-way path between the gut and the brain, the low-grade inflammation originating in the intestine moves to the brain and not vice versa. Moreover, there are 2 barriers in this path between gut and brain, the gut barrier and the $\mathrm{BBB}$, and it is easier for intestinal inflammation to cause disruption of the $\mathrm{BBB}$ and not the other way around.

Another important dietary aspect in MS is hypovitaminosis D, common in people with MS. Fundamentally, this hypovitaminosis may derive from different anomalies: 1) dietary deficiency or low sunlight exposure; 2) altered absorption; 3 ) increased requirement; 4) increased rate of inactivation and excretion; 5) impaired activity of CYP enzymes in vitamin D metabolism (defective synthesis or quick degradation of the active form); 6) lower capability of binding VDR owing to receptor polymorphism or to the competitive binding of bile acids to VDR. Among the above points, the binding of bile acid to VDR connects with gut dysbiosis the low anti-inflammatory activity of vitamin D, whereas the impaired activity of CYP enzymes may be related to diet and molecules present in processed food. Both aspects should be studied in depth.

Finally, the role of vitamin A and the importance of its administration in MS together with vitamin $\mathrm{D}$ should be acknowledged.

We hope that one day we will understand that food is not only pleasure, subsistence, a recipe, a ritual, a way to be together, a symbol of wealth, but that it serves to make us feel better, a means to respect ourselves and to prevent illness. Two thousand years ago, Hippocrates said that food should be our medicine and medicine our food. Nowadays, we have begun to understand that nutrition can really influence our health and we are also beginning to know how it works.

As we started work on the relationship between nutrition and MS 13 years ago nobody believed that such a connection really existed. The same happened with gut microbiota: only 7 years ago was the idea that gut microbiota could have a role in MS considered speculative, but then the role of gut microbiota in neurological disorders became increasingly interesting [11, 44, 124]; for a review see [125]. Now things are changing: more people are taking into consideration the role of dietary intervention in MS [126-128] and the role of gut microbiota in MS.

Most of the work on gut microbiota has been carried out in experimental models. According to Wekerle [129], in MS the pathogenic mechanisms require at least 3 factors: a genetic disposition, a proinflammatory intestinal microbial profile, and the accumulation of autoreactive T cells in GALT.

The presence of a proinflammatory status originating in the intestine has also been demonstrated in humans. As already mentioned above and in Fig. 2, Th17 cells are involved in the pathogenesis of MS and an important role has been assigned to IL-17, which has been found in the active plaques of MS lesions. Recently, the presence of effector Th17 cells has been observed in tissues collected from patients with relapsingremitting MS by esophagogastroduodenoscopy (small intestine). Their presence was linked to specific microbiota changes (lower in Prevotella, higher in Streptococcus) [130].

Unfortunately, the role of nutrition in MS is neglected at present, but among the good news we can say that the National Society for Multiple Sclerosis is responding to the request by patients of MS of more focus on wellness [131] and is creating a wellness group [132]. It will be of the outmost importance that this group creates dietary guidelines to be transmitted to neurologists who are caring for people with MS.

In conclusion, the concept we want to express in this work is that gut microbiota has a strong impact on human health, but it is an ecosystem that evolves "on a leash" by the host [121]. At the end, host health depends on dietary choices and the intake of dietary supplements, in particular vitamins D and $\mathrm{A}$ in case of hypovitaminosis.

Required Author Forms Disclosure forms provided by the authors are available with the online version of this article.

\section{References}

1. Compston A, Coles A. Multiple sclerosis. Lancet 2008; 372:15021517.

2. Yong HY, Yong VW. Stop inflammation and you stop neurodegeneration in MS - YES. Mult Scler 2017; 23:1320-1321.

3. Amato MP, Derfuss T, Hemmer B, et al. 2016 ECTRIMS Focused Workshop Group. Environmental modifiable risk factors for 
multiple sclerosis: report from the 2016 ECTRIMS focused workshop. Mult Scler 2017; 6:1352458516686847.

4. Munger KL, Fitzgerald KC, Freedman MS, et al. No association of multiple sclerosis activity and progression with EBV or tobacco use in BENEFIT. Neurology 2015;85:1694-1701.

5. Napier MD, Poole C, Satten GA, Ashley-Koch A, Marrie RA, Williamson DM. Heavy metals, organic solvents, and multiple sclerosis: An exploratory look at gene-environment interactions. Arch Environ Occup Health 2016; 71:26-34.

6. Munger KL. Childhood obesity is a risk factor for multiple sclerosis. Mult Scler 2013;19:1800.

7. Liu Z, Zhang TT, Yu J, et al. Excess body weight during childhood and adolescence is associated with the risk of multiple sclerosis: a meta-analysis. Neuroepidemiology 2016; 47:103-108.

8. Ascherio A, Munger KL, White R, et al. Vitamin D as an early predictor of multiple sclerosis activity and progression. JAMA Neurol 2014; 71:306-314.

9. Pierrot-Deseilligny C, Souberbielle JC. Vitamin D and multiple sclerosis: an update. Mult Scler Relat Disord 2017; 14:35-45.

10. Riccio P, Rossano R, Liuzzi GM. May diet and dietary supplements improve the wellness of multiple sclerosis patients? A molecular approach. Autoimm Dis 2011; 2010:249842.

11. Riccio $\mathrm{P}$. The molecular basis of nutritional intervention in multiple sclerosis: a narrative review. Complement Ther Med 2011; 19: 228-237.

12. Riccio P, Rossano R. The role of nutrition in multiple sclerosis: a story yet to be written. Rev Esp Esclerosis Multiple 2013; 5:24-37.

13. Riccio P, Rossano R. Nutrition facts in multiple sclerosis. ASN Neuro 2015; 7:1759091414568185.

14. Holick MF. The vitamin D deficiency pandemic: approaches for diagnosis, treatment and prevention. Rev Endocr Metab Disord 2017; 18:153-165.

15. Herieka M, Erridge C. High-fat meal induced postprandial inflammation. Mol Nutr Food Res 2014; 58:136-146.

16. Hedström AK, Olsson T, Alfredsson L. High body mass index before age 20 is associated with increased risk for multiple sclerosis in both men and women. Mult Scler 2012; 18:1334-1336.

17. Wang Y, Kasper LH. The role of microbiome in central nervous system disorders. Brain Behav Immun 2014; 38:1-12.

18. Rea K, Dinan TG, Cryan JF. The microbiome: a key regulator of stress and neuroinflammation. Neurobiol Stress 2016; 4:23-33.

19. Fleck AK, Schuppan D, Wiendl H, Klotz L. Gut-CNS-axis as possibility to modulate inflammatory disease activityimplications for multiple sclerosis. Int J Mol Sci 2017; 18: E1526.

20. Koh A, De Vadder F, Kovatcheva-Datchary P, Bäckhed F. From dietary fiber to host physiology: short-chain fatty acids as key bacterial metabolites. Cell 2016; 165:1332-1345.

21. Tilg H, Moschen AR, Kaser A. Obesity and the microbiota. Gastroenterology 2009; 13:1476-1483.

22. De Filippo C, Cavalieri D, Di Paola M, et al. Impact of diet in shaping gut microbiota revealed by a comparative study in children from Europe and rural Africa. Proc Natl Acad Sci U S A 2010; 107:14691-14696.

23. Lloyd-Price J, Abu-Ali G, Huttenhower C. The healthy human microbiome. Genome Med 2016; 8:51.

24. Bäckhed F, Fraser CM, Ringel Y, et al. Defining a healthy human gut microbiome: current concepts, future directions, and clinical applications. Cell Host Microbe 2012; 12:611-622.

25. David LA, Maurice CF, Carmody RN, et al. Diet rapidly and reproducibly alters the human gut microbiome. Nature 2014; 505:559-563.

26. Le Chatelier E, Nielsen T, Qin J, et al. Richness of human gut microbiome correlates with metabolic markers. Nature 2013; 500:541-546
27. Gorvitovskaia A, Holmes SP, Huse SM. Interpreting Prevotella and Bacteroides as biomarkers of diet and lifestyle. Microbiome 2016; 4:15.

28. Pols TWH, Puchner T, Korkmaz HI, Vos M, Soeters MR, de Vries CJM. Lithocholic acid controls adaptive immune responses by inhibition of Th1 activation through the Vitamin D receptor. PLOS ONE 2017; 12:e176715.

29. Velasquez-Manoff M. Gut microbiome: the peacekeepers. Nature 2015; 518:S3-S11

30. David LA, Materna AC, Friedman J, et al. Host lifestyle affects human microbiota on daily timescales. Genome Biol 2014; 15: R89.

31. Louis P, Flint HJ. Formation of propionate and butyrate by the human colonic microbiota. Environ Microbiol 2017; 19:29-41.

32. Rivière A, Selak M, Lantin D, Leroy F, De Vuyst L. Bifidobacteria and butyrate-producing colon bacteria: Importance and strategies for their stimulation in the human gut. Front Microbiol 2016; 7 : 979.

33. Thorburn AN, Macia L, Mackay CR. Diet, metabolites, and "western-lifestyle" inflammatory diseases. Immunity 2014; 40 833-842.

34. Telesford KM, Yan W, Ochoa-Reparaz J, et al. A commensal symbiotic factor derived from Bacteroides fragilis promotes human CD39(+)Foxp3(+) T cells and Treg function. Gut Microbes 2015; 6:234-242.

35. Quévrain E, Maubert MA, Michon C, et al. Identification of an anti-inflammatory protein from Faecali bacterium prausnitzii, a commensal bacterium deficient in Crohn's disease. Gut 2016; 65: 415-425.

36. Moos WH, Faller DV, Harpp DN, et al. Microbiota and neurological disorders: a gut feeling. Biores Open Access 2016; 5:137-145.

37. Rothhammer V, Mascanfroni ID, Bunse L, et al. Type I interferons and microbial metabolites of tryptophan modulate astrocyte activity and central nervous system inflammation via the aryl hydrocarbon receptor. Nat Med 2016; 22:586-597.

38. Lutgendorff F, Akkermans LM, Söderholm JD. The role of microbiota and probiotics in stress-induced gastro-intestinal damage. Curr Mol Med 2008; 8:282-298.

39. Shen T, Chen X, Li Y, et al. Interleukin-17A exacerbates high-fat diet-induced hepatic steatosis by inhibiting fatty acid $\beta$-oxidation. Biochim Biophys Acta 2017; 1863:1510-1518.

40. Park JH, Jeong SY, Choi AJ, Kim SJ. Lipopolysaccharide directly stimulates Th17 differentiation in vitro modulating phosphorylation of RelB and NF-kB1. Immunol Lett 2015; 165:10-19.

41. Pendyala S, Walker JM, Holt PR. A high-fat diet is associated with endotoxemia that originates from the gut. Gastroenterology 2012; 142:1100-1101.

42. Ochoa-Repáraz J, Kasper LH. The Second Brain: Is the Gut Microbiota a Link Between Obesity and Central Nervous System Disorders? Curr Obes Rep. 2016; 5(1):51-64.

43. Scheperjans F. Can microbiota research change our understanding of neurodegenerative diseases? Neurodegener Dis Manag 2016; 6: 81-85.

44. Berer K, Mues M, Koutrolos M, et al. Commensal microbiota and myelin autoantigen cooperate to trigger autoimmune demyelination. Nature 2011; 479:538-541.

45. Miyake S, Kim S, Suda W, et al. Dysbiosis in the gut microbiota of patients with multiple sclerosis, with a striking depletion of species belonging to Clostridia XIVa and IV Clusters. PLOS ONE 2015; 10:e137429.

46. Chen J, Chia N, Kalari KR, et al. Multiple sclerosis patients have a distinct gut microbiota compared to healthy controls. Sci Rep 2016; 6:28484.

47. Mirza A, Mao-Draayer Y. The gut microbiome and microbial translocation in multiple sclerosis. Clin Immunol 2017. 
48. Scheperjans F, Aho V, Pereira PA, et al. Gut microbiota are related to Parkinson's disease and clinical phenotype. Mov Disord 2015; 30:350-358.

49. Bu XL, Yao XQ, Jiao SS, et al. A study on the association between infectious burden and Alzheimer's disease. Eur J Neurol 2015; 22: $1519-1525$.

50. Bu XL, Wang X, Xiang Y, et al. The association between infectious burden and Parkinson's disease: a case-control study. Parkinsonism Relat Disord 2015; 21:877-881.

51. Buscarinu MC, Cerasoli B, Annibali V, et al. Altered intestinal permeability in patients with relapsing-remitting multiple sclerosis: a pilot study. Mult Scler 2017; 23:442-446.

52. Wekerle H. Brain autoimmunity and intestinal microbiota. 100 Trillion game changers. Trends Immunol 2017; 1379:1-15.

53. Swank RL. Multiple sclerosis; a correlation of its incidence with dietary fat. Am J Med Sci 1950; 220:421-430.

54. Swank RL, Goodwin JW. Review of MS patient survival on a Swank low saturated fat diet. Nutrition 2003; 19:161-165.

55. Rietschel ET, Brade H, Holst $\mathrm{O}$, et al. Bacterial endotoxin: chemical constitution, biological recognition, host response, and immunological detoxification. Curr Top Microbiol Immunol 1996; 216: 39-81.

56. Hwang DH, Kim JA, Lee JY. Mechanisms for the activation of Toll-like receptor $2 / 4$ by saturated fatty acids and inhibition by docosahexaenoic acid. Eur J Pharmacol 2016; 785:24-35.

57. Fritsche KL. The science of fatty acids and inflammation. Adv Nutr 2015; 6:293S-301S.

58. Kleinewietfeld M, Manzel A, Titze J, et al. Sodium chloride drives autoimmune disease by the induction of pathogenicTh17 Cells. Nature 2013; 496:518-522.

59. Riccio P. The proteins of the milk fat globule membrane in the balance. Trends Food Sci Technol 2004; 15:458-461.

60. Choi IY, Lee P, Adany P, et al. In vivo evidence of oxidative stress in brains of patients with progressive multiple sclerosis. Mult Scler 2017.

61. Frischer JM, Stephan Bramow S, Dal-Bianco A, et al. The relation between inflammation and neurodegeneration in multiple sclerosis brains. Brain 2009; 132: 1175-1189.

62. Calder PC. Omega-3 fatty acids and inflammatory processes. Nutrients 2010; 2:355-374.

63. Schmitz G, Ecker J. The opposing effects of n-3 and n-6 fatty acids. Prog Lipid Res 2008; 47:147-155.

64. Fragoso YD, Stoney PN, McCaffery PJ. The evidence for a beneficial role of vitamin A in multiple sclerosis. CNS Drugs 2014; 28:291-299.

65. Huang JK, Jarjour AA, Oumesmar BN, et al. Retinoid X receptor gamma signaling accelerates CNS remyelination. Nat Neurosci 2011; 14:45-53.

66. Qing J. Natural forms of vitamin E: metabolism, antioxidant and anti-inflammatory activities and the role in disease prevention and therapy. Free Radic Biol Med 2014; 72: 76-90.

67. Mastronardi FG, Min W, Wang H, et al. Attenuation of experimental autoimmune encephalomyelitis and non immune demyelination by IFN-beta plus vitamin $\mathrm{B}_{12}$ : treatment to modify notch1/sonic hedgehog balance. J Immunol 2004; 172:6418-6426.

68. Penberthy WT, Tsunoda I. The importance of NAD in multiple sclerosis. Curr Pharm Des 2009; 15:64-99.

69. Fenni S, Hammou H, Astier J, et al. Lycopene and tomato powder supplementation similarly inhibit high-fat diet induced obesity, inflammatory response, and associated metabolic disorders. Mol Nutr Food Res 2017; 61(9).

70. Boosalis MG. The role of selenium in chronic disease. Nutr Clin Pract 2008; 23:152-160.

71. Bredholt M, Frederiksen JL. Zinc in multiple sclerosis: a systematic review and meta-analysis. ASN Neuro 2016; 8: 1759091416651511.
72. Galland G. Diet and inflammation. Nutr Clin Pract 2010; 25:634640.

73. Riccio P, Giovannelli S, Bobba A, et al. Specificity of zinc binding to myelin basic protein. Neurochem Res 1995; 20:1107-1113.

74. Salinthone S, Yadav V, Schillace RV, Bourdette DN, Carr DW. Lipoic acid attenuates inflammation via cAMP and protein kinase A signaling. PLOS ONE 2010; 5:e13058.

75. Takechi R, Pallebage-Gamarallage MM, Lam V, Giles C, Mamo JC. Nutraceutical agents with anti-inflammatory properties prevent dietary saturated-fat induced disturbances in blood-brain barrier function in wild-type mice. J Neuroinflammation 2013; 10:73.

76. Bavarsad Shahripour R, Harrigan MR, Alexandrov AV. Nacetylcysteine (NAC) in neurological disorders: mechanisms of action and therapeutic opportunities. Brain Behav 2014; 4:108122.

77. Visioli F, De La Lastra CA, Andres-Lacueva C, et al. Polyphenols and human health: a prospectus. Crit Rev Food Sci Nutr 2011; 51: 524-546.

78. Gupta C, Prakash D. Phytonutrients as therapeutic agents. J Complement Integr Med 2014; 11:151-169.

79. Vijayakumar TM, Kumar RM, Agrawal A, Dubey GP, Ilango K. Comparative inhibitory potential of selected dietary bioactive polyphenols, phytosterols on CYP3A4 and CYP2D6 with fluorometric high-throughput screening. J Food Sci Technol 2015; 52: 4537-4543.

80. Liuzzi GM, Latronico T, Branà MT, et al. Structure-dependent inhibition of gelatinases by dietary antioxidants in rat astrocytes and sera of multiple sclerosis patients. Neurochem Res 2011; 36: 518-527.

81. Amara F, Berbenni M, Fragni M, et al. Neuroprotection by cocktails of dietary antioxidants under conditions of nerve growth factor deprivation. Oxid Med Cell Longev 2015; 2015:217258.

82. Westfall S, Lomis N, Kahouli I, Dia SY, Singh SP, Prakash S. Microbiome, probiotics and neurodegenerative diseases: deciphering the gut brain axis. Cell Mol Life Sci 2017.

83. Chassaing B, Koren O, Goodrich JK, et al. Dietary emulsifiers impact the mouse gut microbiota promoting colitis and metabolic syndrome. Nature 2015; 519:92-96.

84. Roca-Saavedra P, Mendez-Vilabrille V, Miranda JM, et al. Food additives, contaminants and other minor components: effects on human gut microbiota - a review. J Physiol Biochem 2017.

85. Welzl H, D'Adamo P, Lipp HP. Conditioned taste aversion as a learning and memory paradigm. Behav Brain Res 2001; 125:205213.

86. Weiland TJ, Hadgkiss EJ, Jelinek GA, et al. The association of alcohol consumption and smoking with quality of life, disability and disease activity in an international sample of people with multiple sclerosis. J Neurol Sci 2014; 336:211-219.

87. Allais L, Kerckhof FM, Verschuere S, et al. Chronic cigarette smoke exposure induces microbial and inflammatory shifts and mucin changes in the murine gut. Environ Microbiol 2016; 18: 1352-1363.

88. Hedström AK, Hillert J, Olsson T, Alfredsson L. Alcohol as a modifiable lifestyle factor affecting multiple sclerosis risk. JAMA Neurol 2014; 71:300-305.

89. Samuelson DR, Shellito JE, Maffei VJ, et al. Alcohol-associated intestinal dysbiosis impairs pulmonary host defense against Klebsiella pneumoniae. PLOS Pathog 2017; 13:e1006426.

90. Piccio L, Stark JL, Cross AH. Chronic calorie restriction attenuates experimental autoimmune encephalomyelitis. J Leukocyte Biol 2008; 84:940-948.

91. Choi IY, Piccio L, Childress P, et al. A diet mimicking fasting promotes regeneration and reduces autoimmunity and multiple sclerosis symptoms. Cell Rep 2016; 15:2136-2146.

92. Clauw DJ. Guided graded exercise self-help as a treatment of fatigue in chronic fatigue syndrome. Lancet 2017; 390:335-336. 
93. Gacias M, Casaccia P. Promoting return of function in multiple sclerosis: an integrated approach. Mult Scler Relat Disord 2013; 2(4).

94. Mokhtarzade M, Ranjbar R, Majdinasab N, Patel D, Molanouri Shamsi M. Effect of aerobic interval training on serum IL-10, $\mathrm{TNF} \alpha$, and adipokines levels in women with multiple sclerosis: possible relations with fatigue and quality of life. Endocrine 2017; 57:262-271.

95. Lunde LK, Skare Ø, Aass HC, et al. Physical activity initiated by employer induces improvements in a novel set of biomarkers of inflammation: an 8-week follow-up study. Eur J Appl Physiol 2017; 117:521-532.

96. Petriz BA, Castro AP, Almeida JA, et al. Exercise induction of gut microbiota modifications in obese, non-obese and hypertensive rats. BMC Genomics. 2014;15:511.

97. Monda V, Villano I, Messina A, et al. Exercise modifies the gut microbiota with positive health effects. Oxid Med Cell Longev 2017; 2017:3831972.

98. Farinotti M, Vacchi L, Simi S, Di Pietrantonj C, Brait L, Filippini G. Dietary interventions for multiple sclerosis. Cochrane Database Syst Rev 2012; 12:CD004192.

99. Kousmine C. Inflammation, allergie et cancer. Int Arch Allergy Appl Immunol 1956; 8:207-217.

100. Swank RL. Multiple sclerosis: twenty years on low fat diet. Arch Neurol 1970; 23:460-474.

101. Bisht B, Darling WG, Grossmann RE, et al. A multimodal intervention for patients with secondary progressive multiple sclerosis: feasibility and effect on fatigue. J Altern Complement Med 2014; 20:347-355.

102. Ostan R, Lanzarini C, Pini E, et al. Inflammaging and cancer: a challenge for the Mediterranean diet. Nutrients 2015; 7:25892621.

103. McDougall J, Thomas LE, McDougall C, et al. Effects of 7 days on an ad libitum low-fat vegan diet: the McDougall Program cohort. Nutr J 2014; 13:99.

104. El-Chammas K, Danner E. Gluten-free diet in nonceliac disease. Nutr Clin Pract 2011; 26:294-299.

105. Riccio P, Rossano R, Larocca M, et al. Anti-inflammatory nutritional intervention in patients with relapsing-remitting and primary-progressive multiple sclerosis: a pilot study. Exp Biol Med (Maywood) 2016; 24:620-635.

106. Rossano R, Larocca M, Riviello L, et al. Heterogeneity of serum gelatinases MMP-2 and MMP-9 isoforms and charge variants. J Cell Mol Med 2014; 18:242-252.

107. Ascherio A, Munger KL. Epidemiology of multiple sclerosis: from risk factors to prevention - an update. Semin Neurol 2016; 36:103-114.

108. Charalambidou E., Pantzaris M., Patrikios I. Multiple sclerosis in Cyprus: a fourteen year (2000-2014) epidemiological study. Am J Epidemiol Infect Dis 2016; 4:1-9.

109. Massacesi L, Abbamondi AL, Giorgi C, Sarlo F, Lolli F, Amaducci L. Suppression of experimental allergic encephalomyelitis by retinoic acid. J Neurol Sci 1987; 80:55-64.

110. Al-Daghri NM, Guerini FR, Al-Attas OS, et al. Vitamin D receptor gene polymorphisms are associated with obesity and inflammosome activity. PLOS ONE 2014; 9:e102141.

111. Polidoro L, Properzi G, Marampon F, et al. Vitamin D protects human endothelial cells from $\mathrm{H}_{2} \mathrm{O}_{2}$ oxidant injury through the Mek/Erk-Sirt1 axis activation. J Cardiovasc Transl Res 2013; 6: 221-231.
112. Zhao $\mathrm{H}$, Zhang $\mathrm{H}$, Wu H, et al. Protective role of $1,25(\mathrm{OH}) 2$ vitamin D3 in the mucosal injury and epithelial barrier disruption in DSS-induced acute colitis in mice. BMC Gastroenterol 2012; 12:57.

113. Jin $\mathrm{D}, \mathrm{Wu} \mathrm{S}$, Zhang YG, et al. Lack of vitamin D receptor causes dysbiosis and changes the functions of the murine intestinal microbiome. Clin Ther 2015; 37:996-1009.

114. Sun J. VDR/vitamin D receptor regulates autophagic activity through ATG16L1. Autophagy 2016; 12:1057-1058.

115. Sun J. The Role of vitamin D and vitamin D receptors in colon cancer. Clin Transl Gastroenterol 2017; 8:e103.

116. Pantzaris MC, Loukaides GN, Ntzani EE, Patrikios IS. A novel oral nutraceutical formula of omega-3 and omega- 6 fatty acids with vitamins (PLP10) in relapsing remitting multiple sclerosis: a randomised, double-blind, placebo-controlled proof-of-concept clinical trial. BMJ Open 2013; 3:e02170.

117. Gupta A, Khanna S. Fecal microbiota transplantation. JAMA 2017; 318: 102.

118. Callaway E. Fish live longer on 'young poo'. Nature 2017; 544: 147.

119. Smith P, Willemsen D, Popkes ML, et al. Regulation of life span by the gut microbiota in the short-lived African turquoise killifish. Elife 2017;6:e27014.

120. Bojanova DP, Bordenstein SR. Fecal transplants: what is being transferred? PLOS Biol. 2016; 14:e1002503.

121. Foster KR, Schluter J, Coyte KZ, Rakoff-Nahoum S. The evolution of the host microbiome as an ecosystem on a leash. Nature 2017; 548:43-51.

122. Sonnenburg JL, Bäckhed F. Diet-microbiota interactions as moderators of human metabolism. Nature 2016; 535:56-64.

123. Ochoa-Repáraz J, Magori K, Kasper LH. The chicken or the egg dilemma: intestinal dysbiosis in multiple sclerosis. Ann Transl Med 2017; 5:145

124. Ochoa-Repáraz J, Mielcarz DW, Begum-Haque S, Kasper LH. Gut, bugs, and brain: role of commensal bacteria in the control of central nervous system disease. Ann Neurol 2011; 69:240-247.

125. Fernández Ó, Álvarez-Cermeño JC, Arroyo-González R, et al. Review of the novelties presented at the $27^{\text {th }}$ Congress of the European Committee for Treatment and Research in Multiple Sclerosis (ECTRIMS). Rev Neurol 2012; 54:677-691.

126. Esposito S, Bonavita S, Sparaco M, Gallo A, Tedeschi G. The role of diet in multiple sclerosis: a review. Nutr Neurosci 2017; 1-14.

127. Altowaijri G, Fryman A, Yadav V. Dietary interventions and multiple sclerosis. Curr Neurol Neurosci Rep 2017; 17:28.

128. Bagur MJ, Murcia MA, Jiménez-Monreal AM, et al. Influence of diet in multiple sclerosis: a systematic review. Adv Nutr 2017; 8: 463-472.

129. Wekerle $\mathrm{H}$. The gut-brain connection: triggering of brain autoimmune disease by commensal gut bacteria. Rheumatology (Oxford) 2016; 55:68-75.

130. Cosorich I, Dalla-Costa G, Sorini C, et al. High frequency of intestinal TH17 cells correlates with microbiota alterations and disease activity in multiple sclerosis. Sci Adv 2017; 3:e1700492.

131. Dunn M, Bhargava P, Kalb R. Your patients with multiple sclerosis have set wellness as a high priority - and the national multiple sclerosis society is responding. US Neurology 2015; 11:80-86.

132. Motl RW, Mowry EM, Ehde DM, et al. Wellness and multiple sclerosis: the national MS society establishes a wellness research working group and research priorities. Mult Scler 2017. 